\title{
Comparación de la eficiencia científica entre Colombia y México a través de indicadores relativos de producción y calidad científica
}

\author{
Nubia Gómez-Velasco*, Ana Jiménez-González*, Julieth Rodríguez-Gutiérrez*, Mauricio Romero-Torres** \\ * Escuela de Matemáticas y Estadística, Facultad de Ciencias, Universidad Pedagógica y Tecnológica de Colombia UPTC \\ Correo-e: nubia.gomez@uptc.edu.co | ORCID iD: https://orcid.org/0000-0001-7745-1721 \\ Correo-e: ana.jimenez@uptc.edu.co | ORCID iD: https://orcid.org/0000-0003-0063-943X \\ Correo-e: juliethkatherine.rodriguez@uptc.edu.co | ORCID iD: https://orcid.org/0000-0001-9845-039X \\ ** Unidad Nacional para la Gestión del Riesgo de Desastres (UNGRD), Subdirección para el Conocimiento del Riesgo, \\ Bogotá, Colombia \\ Correo-e: mauricio.romero@gestiondelriesgo.gov.co | ORCID iD: https://orcid.org/0000-0003-3282-0372
}

Recibido: 29-01-2019; 2a versión: 20-05-2019; Aceptado: 06-06-2019.

Cómo citar este artículo/Citation: Gómez-Velasco, N., Jiménez-González, A., Rodríguez-Gutiérrez, J., Romero-Torres, M. (2020). Comparación de la eficiencia científica entre Colombia y México a través de indicadores relativos de producción y calidad científica. Revista Española de Documentación Científica, 43 (2), e262. https://doi.org/10.3989/redc.2020.2.1644

Resumen: Con indicadores relativos analizamos la dinámica de la producción y calidad científica de Colombia y México en el área de Ciencias Sociales (CS). Entre 2006 a 2017 estimamos la producción científica, la inversión en I+D, la cantidad de doctores graduados, la calidad científica por medio del porcentaje de revistas Q1 con autorías nacionales y el porcentaje de citaciones en revistas prestigiosas visibles en Scopus. Encontramos que la inversión en I+D, la producción científica y los graduados doctores de México son superiores a Colombia; sin embargo, Colombia superó a México en la eficiencia de la investigación. Ni Colombia ni México reportaron revistas en Q1 para CS, lo cual reflejó sus bajos niveles de indicadores de citación. Mostramos que el uso de indicadores relativos complementa al análisis bibliométrico tradicional.

Palabras clave: producción científica; ciencias sociales; indicadores relativos; inversión I+D; eficiencia científica.

\section{Comparison of the scientific efficiency between Colombia and Mexico through relative indicators of production and scientific quality}

\begin{abstract}
We analyzed the dynamics of Colombian and Mexican scientific production, and quality in Social Sciences (SS), using relative indicators. For the period of time between 2006 and 2017, we estimated the scientific production, investment in R\&D and the number of graduate doctors, as well as the percentages of the scientific quality of Q1 journals with national authorship and their citations in prestigious journals visible in Scopus. We found that the R\&D investment, scientific production, and the number of graduate doctors in Mexico is higher than Colombia. However, Colombia surpassed Mexico in research productivity. Neither Colombia nor Mexico reported journals in Q1 for SS, reflecting the low levels of citation indicators. We show that the use of relative indicators complements the conventional bibliometric analysis.
\end{abstract}

Keywords: scientific production; social sciences and humanities; relative indicators; research and development investment; scientific efficiency.

Copyright: ( 2020 CSIC. Este es un artículo de acceso abierto distribuido bajo los términos de la licencia de uso y distribución Creative Commons Reconocimiento 4.0 Internacional (CC BY 4.0). 


\section{INTRODUCCIÓN}

La inversión en Ciencia y Tecnología (CyT) se distribuye globalmente de manera desigual. En cada país las prioridades de inversión para СуТ cambian de acuerdo con las restricciones presupuestales, las fortalezas históricas de los equipos de investigación y las demandas de la industria. Los países con economías basadas en conocimiento tienden a generar ritmos similares de crecimiento económico con una continua acumulación y transmisión del conocimiento que impacta positivamente su desarrollo socioeconómico (Mansell y Steinmueller 2000; Teitel, 1994); premisa que revisaremos en Colombia y México a partir de su producción y calidad científica. Se ha planteado la existencia de relaciones causales en el largo plazo entre innovación y crecimiento económico (Pradhan y otros, 2017), y se predice que aumentar la inversión en CyT para aumentar la capacidad productiva de los bienes de capital, generaría un desarrollo científico local. Por lo anterior, la relación entre crecimiento económico y el desarrollo en CyT es un tema de creciente interés entre países con economías y capacidades científicas equivalentes (Van den Berg, 2016).

En América Latina los resultados de CyT no son alentadores. En comparación con países desarrollados, por décadas ha existido un bajo nivel de inversión en investigación y desarrollo (I+D), capital humano e infraestructura tecnológica, y existe un bajo desarrollo de la economía basada en el conocimiento (Casas, 2004). Así mismo, en América Latina la productividad científica se concentra en las universidades, aspecto que en el marco de la teoría de las organizaciones implica una similitud del sistema organizacional universitario con el sistema organizacional empresarial (Weick y otros, 2009). Se ha planteado que la producción científica engloba múltiples procesos y variables socioeconómicas, y refleja la capacidad científica de un país a escala de personas, asociaciones y consumidores (Witter, 1997).

Colombia y México, junto con Brasil, Chile y Argentina, poseen las mayores economías de América Latina. Colombia y México tienen a pesar de los recientes problemas de narcotráfico y violencia, un sistema democrático estable, problemáticas similares de seguridad social y una preocupación en afianzar relaciones con países desarrollados como Estados Unidos (Ardila, 2015). Colombia y México poseen diferencias en su tasa de crecimiento promedio, en la mano de obra por hora trabajada, pero similitudes en el bajo desempeño de los Factores Totales de Productividad que explican el crecimiento económico de ambos países. Estas diferencias no han limitado la integración económica y comercial, que se traducen en tratados de libre co- mercio y mecanismos de integración regional como la Alianza del Pacífico (Hofman y otros, 2017). Es lógico que México genere una mayor productividad científica que Colombia -y que la mayoría de los países latinoamericanos a excepción de Brasil- ya que cuenta con mayor inversión en $I+D$ y mayor número de investigadores. Entre el 2006 a 2013, México contribuyó con el $16,1 \%$ de las publicaciones científicas en América Latina, mientras que Colombia aportó el 4\% (RICYT, 2017).

Comprender la relación entre crecimiento económico y desarrollo científico-tecnológico requiere mejores indicadores de CyТ (Teitel, 1985). Esta necesidad es aún más crítica en países latinoamericanos con contextos, recursos y políticas de investigación diferentes de los países desarrollados (Aguado y Becerril, 2016; Morales Gaitán y Aguado López, 2010). Para evitar comparaciones con indicadores de ciencia de países anglosajones y asiáticos que generan una distorsión debido a las desventajas del sistema de ciencia latinoamericano, son necesarios indicadores y umbrales para evaluar la ciencia regional en su propio contexto. Así mismo, se han criticado los sesgos de las bases de datos internacionales para generar indicadores de CyT. Estos sesgos incluyen la precisión en los sistemas de clasificación (Wang y Waltman, 2016), la subrepresentación de la ciencia regional latinoamericana (Aguado y Becerril, 2016), y las acusaciones de oligopolio de algunas editoriales comerciales (Larivière y otros, 2015).

Una alternativa para comparar resultados de CyT es a través de indicadores relativos. Este tipo de indicadores -que se expresan en valores obtenidos por cocientes o porcentajes- se emplean para comparar las instituciones dentro y entre entre países (Villaveces y otros, 2007). Estas comparaciones revelan las diferencias en eficiencia de los sistemas de CyT y generan valores de referencia sobre los resultados nacionales de producción científica y visibilidad (Chinchilla-Rodríguez y otros, 2012; Chinchilla-Rodríguez y otros, 2016; Gómez y otros, 2014; Rodríguez y otros, 2017).

Para comprender la dinámica de la producción científica entre Colombia y México, analizamos dos conjuntos de variables a partir de ocho indicadores relativos. Primero, analizamos indicadores de producción científica como la relación entre la producción científica por cada millón de dólares invertido en I+D, la producción por cantidad de doctores graduados en el área de ciencias sociales, y la citación según antigüedad de la publicación. Segundo, analizamos indicadores de alta calidad como el porcentaje de revistas Q1 con autorías nacionales, y el porcentaje de citaciones en revistas con altos valores SCImago Journal Rank (SJR). 


\section{METODOLOGÍA}

Para medir la producción científica de Colombia y México entre los años 2007 a 2017 se extrajeron datos desde Scopus (www.scopus.com). Se tomaron como criterios delimitadores de búsqueda: país (Colombia y México), años (2007-2017), área de conocimiento/Subject-Area (Ciencias Sociales) y tipo de documento (artículos). Los resultados de las búsquedas se tabularon en Excel, y se utilizó el programa VantagePoint-VPEI (versión 11, Search Technology) para depurar y normalizar los nombres de autores, revistas e instituciones. Los datos tabulados también incluyeron variables como el año de publicación, idioma, revista y número de citas por artículo. A partir de esta información se calculó el total de artículos publicados por cada país (F) y el total de citaciones recibidas (G, Fig. 1A). Para determinar el total de revistas del cuartil uno (Q1), se extrajeron y tabularon datos desde SCImago Journal Rank (SCImago, 2007) para los años 2007 a 2017. A partir del conjunto de revistas Q1 (A, Fig. 1A), se estimó el conjunto de revistas TOP (B), la cantidad de artículos (D) y de citaciones (E) en revistas TOP (Fig. 1A).

Los datos de formación doctoral y el número de investigadores se tomaron de la Red de Indicadores de Ciencia y Tecnología Iberoamericana e Interamericana (RICYT) (http://www.ricyt.org/). En todas las fuentes de datos se utilizaron los periodos más actualizados posibles (consulta en febrero de 2019). En ausencia de información de la RICYT para el año 2017, se ajustaron regresiones polinómicas para predecir el valor de inversión en millones de dólares en I+D y para predecir el número de doctores en Ciencias Sociales. La predicción de la inversión en $\mathrm{I}+\mathrm{D}$ para Colombia y México tuvo un ajuste alto $\left(R^{2}=0,87\right.$ y 0,81$)$, respectivamente. La predicción del número de doctores también mostró un ajuste alto para Colombia y México $\left(R^{2}=0,92\right.$ y $=0,93)$, respectivamente. A partir del conjunto de datos tabulados se calcularon los siguientes ocho indicadores relativos ( $R 1$ a R8).

Producción de artículos en el área de Ciencias Sociales (CS) respecto a la inversión en I+D (R1): cociente entre la cantidad de artículos con afiliación a un país en el área de CS y los recursos financieros provenientes del presupuesto nacional para I+D en millones de dólares corrientes, calculado para cada año en el periodo de estudio.

Producción de artículos en CS respecto al total de doctores graduados en CS (R2): cociente entre la producción científica representada por la cantidad de artículos y el total de doctores graduados en el área de Ciencias Sociales-CS. Según Acosta y Celis (2014) la formación doctoral impulsa la in- vestigación de calidad y por ende las publicaciones científicas en un país. Se siguió la metodología de Spinak (1998), Sancho (1990) y Pérez y Martínez (2014) para medir indicadores como el número de artículos publicados por país, su distribución por año, su tasa de crecimiento, las revistas principales y los valores de citación.

Impacto de citación normalizado (R3): número de citaciones recibidas por cada país, comparado con el promedio mundial de citaciones recibidas por publicación del mismo tipo, año y categoría. El impacto de citación normalizado (NI) se calculó a través del Item oriented field normalized citation score average (Bornmann y Haunschild, 2016). Los porcentajes muestran las relaciones entre el impacto científico promedio del país y el conjunto promedio mundial con una puntuación de 1 ; es decir, una puntuación de NI de 0.8 significa que la institución es citada un $20 \%$ por debajo del promedio mundial y un valor de 1.3 significa que la institución es citada un $30 \%$ superior a la media del mundo.

Publicaciones de alta calidad (R4): porcentaje de documentos en CS que publica un país en las revistas más influyentes del mundo (denominadas revistas TOP); es decir, las revistas clasificadas en el $10 \%$ superior de Q1 del indicador SJR (Miguel y otros, 2011). Este indicador se obtiene a partir del cociente entre el número de artículos que se ubican en el $10 \%$ de las revistas Q1 y el total de artículos publicados con afiliación al país para el año particular de estudio, así: publicaciones de alta calidad $(\mathrm{R} 4=\mathrm{D} / \mathrm{F}$, Fig. 1A).

Revistas de alta calidad (R5): cociente entre la cantidad de revistas TOP en las que publica los autores de un país y el total de revistas TOP a nivel mundial (Torres-Salinas, y otros, 2011) $(R 5=C / B$, Fig. 1A).

Promedio de artículos por revistas TOP (R6): cociente entre la cantidad de artículos con afiliación del país publicados en revistas TOP y el total de revistas TOP en las que publica los autores de un país (Torres-Salinas, y otros, 2011) (R6 = D/C, Fig. 1A).

Porcentaje de citaciones en revistas TOP (R7): cociente entre total de citaciones de los artículos de los autores de un país que se encuentran en las revistas TOP y el total de citaciones recibidas por un país (Torres-Salinas, y otros, 2011) $(R 7=E / G$, Fig. 1A).

El indicador nacional TOP para cada país (R8), se calculó como el cociente entre el número de artículos que se ubican en el $10 \%$ de las revistas del país de mayor SJR (sin importar cuartil) y el total de artículos publicados con afiliación del país para cada año. 


\section{RESULTADOS}

Entre los años 2006 a 2017 para el área de Ciencias Sociales-CS encontramos en total 7275 artículos científicos con afiliación institucional colombiana y 13249 artículos con afiliación institucional mexicana (Fig. 1). En ambos países se incrementó la producción de artículos en el tiempo; sin embargo, el porcentaje de artículos que recibieron al menos una citación decreció desde un $\sim 80 \%$ a un $\sim 40 \%$ (Fig. 1B). Los principales idiomas de publicación fueron el inglés y el español, con un $69,1 \%$ y un $29,4 \%$ para Colombia, y un $77,3 \%$ y un $21,8 \%$ para México, respectivamente. Los porcentajes restantes se publicaron en otros idiomas como francés, portugués y alemán. Respecto al ingreso de las revistas locales en Scopus, Colombia aumentó en el Scimago Journal Rank de 2 revistas en 2006 a 33 revistas en 2017, con una tasa promedio de crecimiento interanual de $26,3 \%$, mientras que México aumentó de 6 a 34 revistas, con una tasa promedio de 15,6\%. La contribución de Colombia respecto al número total de revistas en SJR aumentó de 0,06\% a 0,58\%, y en México de 0,17\% a 0,59\%.

Entre el 2006 a 2017, los artículos en el área de Ciencias Sociales representaron el $21 \%$ y $17,4 \%$ de la producción científica total de Colombia y México, respectivamente. A nivel latinoamericano, la producción de Colombia representó el 8,1\%, mientras que la de México, el 14,7\%. A nivel mundial, en el área de Ciencias Sociales Colombia aportó el 0,32\% y México el $0,58 \%$. Determinamos que el promedio de artículos publicados en CS para Colombia fue de 605,9 artículos por año y en México de 1104 artículos por año (coeficiente de variación de 63,9\% y 41,7\%, respectivamente). En el periodo de estudio, la tasa de crecimiento promedio anual para Colombia fue del $26,1 \%$ mientras que para México fue del $11 \%$.

Establecimos que la inversión en $\mathrm{I}+\mathrm{D}$ respecto al PIB en Colombia fue de $0,15 \%$ en el año 2006 y de $0,28 \%$ en el año 2017. México tuvo una inversión para I+D respecto al PIB de 0,37\% en el año 2006 y de $0,61 \%$ en el año 2017. Para el indicador R1 determinamos número de artículos de CS visibles en Scopus, respecto a la inversión en I+D, Fig. 1 C. En 2017, Colombia publicó 1,20 artículos en CS por cada millón de dólares invertidos y México publicó 0,64, lo cual significa que Colombia presentó un R1 casi dos veces mayor que México.

Para el indicador R2 (relación entre producción de artículos en CS y el total de doctores graduados en el área de CS, Fig. 1C), establecimos que en 2006 Colombia graduó 23 doctores y México 689 (rela-

Figura 1. Indicadores de productividad científica en Ciencias Sociales para Colombia y México
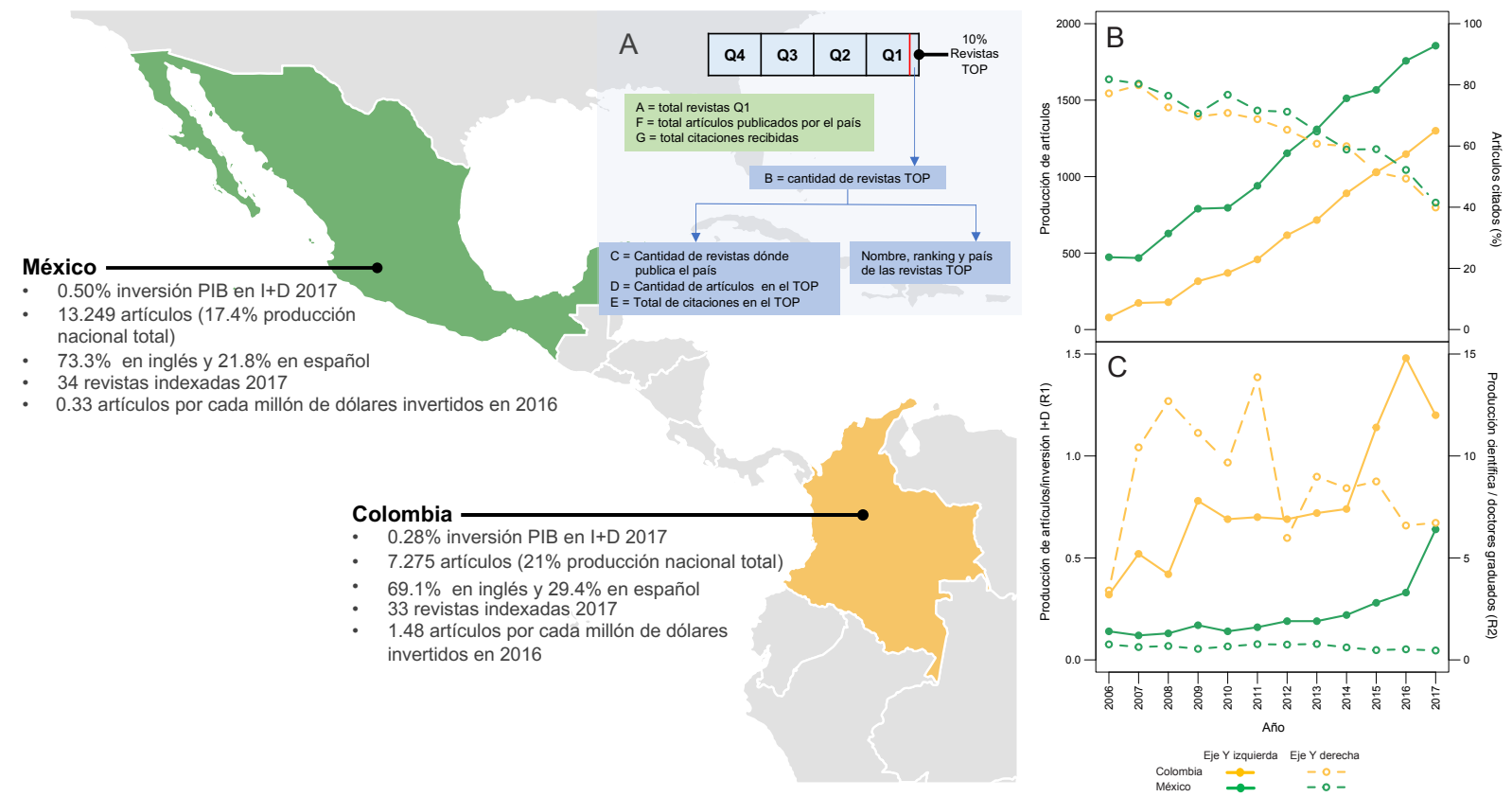

(A) Principales indicadores sobre producción de artículos científicos y variables para el cálculo de revistas TOP. (B) La producción de artículos aumentó en el tiempo, pero el porcentaje de artículos citados al menos una vez disminuyó. (C) Existió una tendencia creciente de la producción de artículos respecto a la inversión en I+D (R1). La producción científica respecto a los doctores graduados (R2) fue estable en México y altamente variable en Colombia. 
ción 1:30). En 2017, Colombia graduó 188 doctores y México 4004 (relación 1:21), lo cual indica una disminución en la brecha de recurso humano con alto nivel de formación. Para R2 Colombia tuvo valores mayores que México en todos los años. Por ejemplo, en 2017 Colombia registró una relación de 6,7 artículos por doctor graduado, mientras que México mostró una relación de 0,46 artículos por doctor graduado (Fig. 1C). Sobre el indicador R3 (impacto de citación normalizado) para el periodo de estudio establecimos que Colombia obtuvo un valor de 0,51 y México de 0,56, valores que indican que las publicaciones se citaron en $49 \%$ y $44 \%$ por debajo del promedio mundial, respectivamente.

Respecto a las publicaciones de alta calidad (R4, Fig. 2A), los dos países reportaron porcentajes de publicación similares en las revistas TOP. Se observó en el periodo de estudio que Colombia publicó en promedio el $1,8 \%$ y México el $1,7 \%$ en revistas TOP (10\% superior de Q1). Sobre las revistas de alta calidad (R5), encontramos que los autores con afiliación a instituciones mexicanas publicaron sus artículos en una mayor cantidad de revistas TOP respecto a Colombia (Fig. 2B) y desde 2006 tanto México como Colombia aumentaron el número de artículos en revistas TOP; es decir, publican cada vez más en revistas TOP diferentes. México entre 2006 a 2011 tuvo un promedio de 7,3\% artículos en revistas TOP y aumentó a un promedio 13,6\% entre 2012 a 2017, mientras que Colombia pasó de $3,4 \%$ a $9,1 \%$ en el mismo periodo.
Sobre el promedio de artículos por revistas TOP (R6), Colombia tuvo valores entre 1 y 1,6 y México valores entre 1 y 1,7 (Fig. 2C). Es decir, que tanto Colombia como México publicaron por año en promedio un artículo en las revistas TOP. En los dos países, el mayor crecimiento de artículos publicados en revistas TOP ocurrió a partir del año 2012. Entre 2006 y 2011 Colombia publicó en promedio 3,8 artículos por año, mientras que México 9 artículos. Entre 2012 y 2017 Colombia publicó en promedio 17,5 artículos por año y México 28,5 artículos.

Sobre el porcentaje de citaciones en revistas TOP (R7), los dos países reportaron un aumento en las citaciones de los artículos publicados en revistas TOP (Fig. 2D). Del total de citaciones que recibieron los artículos publicados por autores de afiliación a cada país, el $11,7 \%$ de Colombia y el $8,6 \%$ de México corresponden a artículos publicados en revistas TOP. En el periodo 2013 a 2016, Colombia mostró valores superiores a México con un promedio de 529,8 citaciones por artículo, frente a 486,2 citaciones.

Así mismo, para los autores con afiliación colombiana establecimos que en el periodo de 12 años, la revista Nature Climate Change reportó la mayor cantidad de citaciones por publicación (1293 citaciones con 9 artículos). Para autores mexicanos la revista World Development reportó 1429 citaciones y 37 artículos. El $50 \%$ de las citas para Colombia se concentra en el $5,8 \%$ de las revistas

Figura 2. Indicadores de calidad de la productividad en Colombia y México en el área de CS
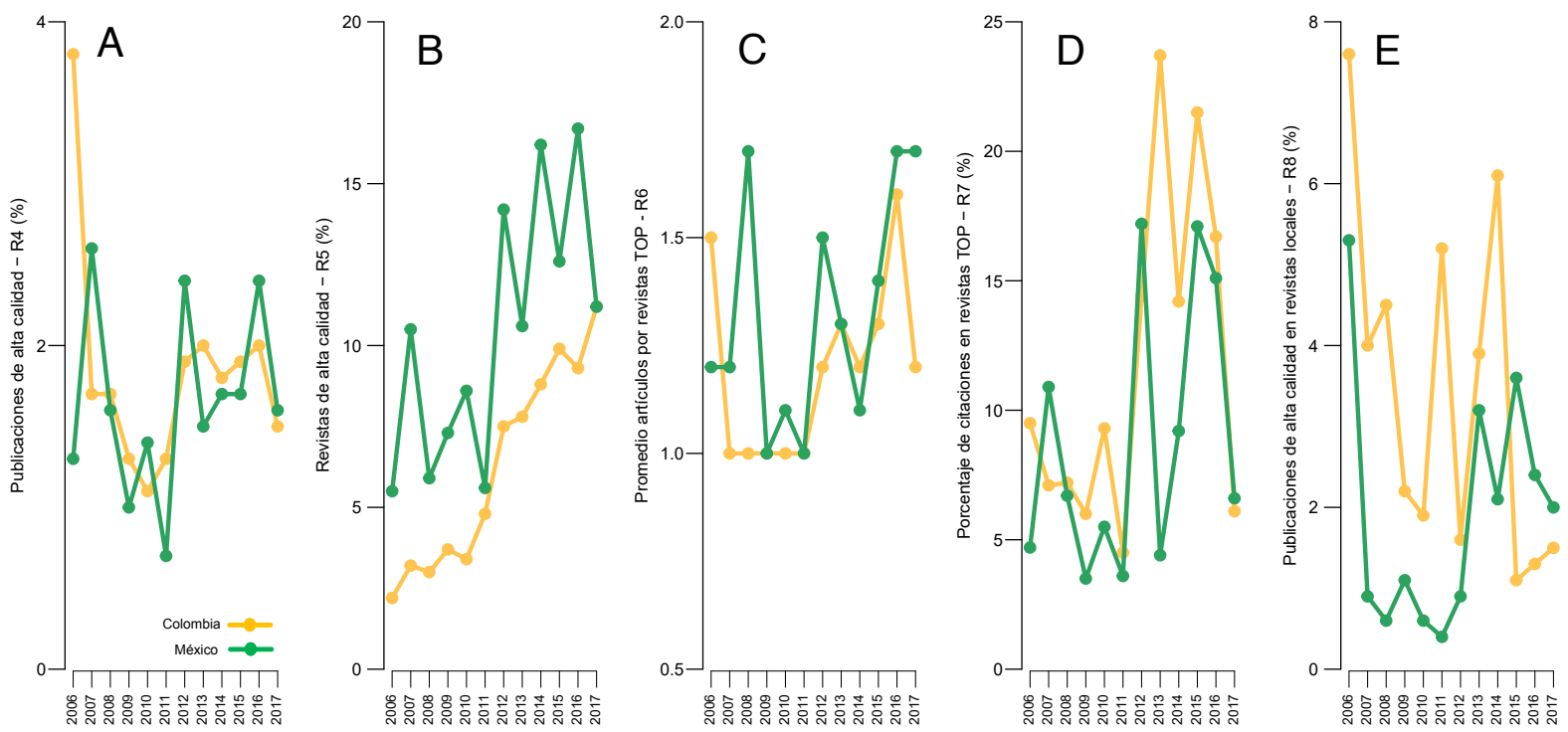

(A) Publicaciones de alta calidad - R4, (B) revistas de alta calidad - R5, (C) promedio de artículos por revistas TOP - R6, (D) porcentaje de citaciones en revistas TOP - R7, y (E) publicaciones de alta calidad en revistas locales - R8. Las revistas TOP se definen como las clasificadas en el $10 \%$ superior de Q1 del indicador SJR. 
(distribuida en el $29 \%$ de los artículos), y para México, el mismo porcentaje de citación se concentra en el $4,5 \%$ de las revistas (distribuida en el $27,5 \%$ de los artículos).

En relación con el porcentaje de artículos publicados en las revistas locales de mayor influencia (R8), hasta el año 2015 Colombia reportó porcentajes mayores que México, es decir, del total de la producción nacional, Colombia publicó más artículos en las revistas locales que México (Fig. 2E). También observamos que este porcentaje es superior al porcentaje de artículos en revistas TOP mundial (R4), para Colombia en todo el periodo de estudio, y para México hasta el año 2012. Se destaca que los investigadores con afiliación colombiana y mexicana tienen publicaciones en revistas TOP nacionales, precisando que en ambos países cuentan con pocas revistas TOP editadas en el país. Por último, establecimos que ninguno de los dos países cuenta con revistas locales en Q1 (i.e., ninguna revista local se encuentra en el TOP mundial).

\section{DISCUSIÓN}

Analizamos ocho indicadores relativos para comprender la dinámica de la producción científica entre Colombia y México entre 2006 y 2017. Encontramos que en 2017 México tuvo casi el doble de producción científica y el doble de inversión en I+D respecto al PIB que Colombia (Fig. 1). Estos valores fueron inferiores a la media de inversión en América Latina y significativamente inferiores al promedio mundial (RICYT, 2017). Al relacionar la inversión en millones de dólares con el número de publicaciones científicas en CS, México produjo menos de un artículo por millón de dólares invertido. Colombia publicó un artículo por cada millón de dólares invertidos solo a partir del año 2015. La hipótesis es que el número de publicaciones científicas por millón de dólares invertido puede doblarse al incluir otras bases bibliográficas regionales como SciELO, Redalyc y Dialnet. Aunque la producción científica visible en Scopus se emplea regularmente como fuente de información, se desconoce qué porcentaje de la producción total colombiana y mexicana en CS cubre Scopus. A pesar de estas críticas, Scopus es por ahora la base de datos bibliográfica con mejor cobertura y herramientas para el análisis bibliométrico de la región. Para comparar su cobertura con Scopus, en el futuro deben probarse nuevos productos como Dimensions y Crossref (Harzing, 2019).

En 2017 México graduó 21 veces más doctores que Colombia en CS, pero Colombia presentó casi 15 veces más producción que México por doctor graduado en CS. Según la RICYT (2017), las dos áreas de conocimiento de la OCDE con mayor porcentaje de doctores graduados se encuentran en Ciencias Sociales y Humanidades, $23,2 \%$ y $22,2 \%$ para Colombia, $39,2 \%$ y $22,9 \%$ para México. A nivel de América Latina y el Caribe, los porcentajes de doctores graduados en el área de Ciencias Sociales y Humanidades fue del $25,6 \%$ y $23,5 \%$ (RICYT, 2017), lo que muestra que México supera el porcentaje latinoamericano en CS. La tendencia creciente en el tiempo de la producción científica y de investigadores por cada millón de habitantes es una tendencia global (UNESCO, 2015). Para el caso latinoamericano, Brasil y México son líderes en la región en ciencia y tecnología (RICYT, 2017); sin embargo, relacionar la producción científica de un país con la inversión en I+D y el tamaño de su fuerza de trabajo científica, da una indicación complementaria de la eficiencia de su investigación. Mostramos que Colombia tuvo una eficiencia de producción científica mayor que México en el área de Ciencias Sociales.

La poca visibilidad e impacto internacional de las revistas colombianas y mexicanas en Scopus se reflejó tanto en los bajos niveles de los indicadores de citas, como en la ausencia de revistas en el cuartil 1. Este fenómeno de baja citación concuerda con otras regiones como Asia y África, problema de las revistas científicas denominado "sur global" (Rogel-Salazar y otros, 2017). A pesar de los bajos niveles de citación, se estima que Colombia es uno de los países en el mundo con mayor acceso abierto a sus artículos ( $60 \%$, Van Noorden, 2019), aspecto que debería reconocerse en las clasificaciones internacionales de revistas. Entre 2006 a 2017, la producción científica de Colombia y México tuvo un aumento notable. La tasa de crecimiento interanual para Colombia fue mayor que la tasa de México, lo cual se explica porque en este periodo Scopus indexó más revistas de Colombia. Actualmente no hay una gran diferencia en el número de revistas, ya que en 2017 México reportó una revista indexada más que Colombia.

En los indicadores de productividad científica Colombia y México mostraron aspectos positivos como la tendencia de crecimiento en inversión en $\mathrm{I}+\mathrm{D}$, el crecimiento en número de publicaciones e investigadores y la vinculación de mayor número de revistas en bases bibliográficas internacionales. Sin embargo, existió un alto porcentaje de artículos sin citas y un bajo porcentaje de publicaciones en revistas de impacto. No sólo una baja inversión en I+D (respecto a países desarrollados) explica este bajo impacto. Las condiciones laborales de Latinoamérica como la concentración de los investigadores/profesores en las universi- 
dades (poca producción científica de la industria), y el número alto de horas docencia/investigación, impactan negativamente en el desempeño en investigación. Estas condiciones motivan a repensar dos aspectos. Primero, para qué y para quienes se produce el conocimiento en estos países. Segundo, con qué finalidad se motiva a sus investigadores en el área de Ciencias Sociales a publicar en revistas internacionales con preferencia sobre sus propias revistas locales.

Observamos que no fue favorable la citación de los artículos publicados en español en el área de Ciencias Sociales. Aunque localmente las comunidades de investigadores se esfuerzan por posicionar sus revistas locales, aquellos investigadores que publican en revistas TOP internacionales se benefician de mayores niveles de citación y visibilidad respecto a las revistas locales con mayor prestigio. Las tendencias de crecimiento del número de artículos latinoamericanos en revistas internacionales (y decrecimiento de las locales) de los últimos años también coinciden con la promoción de las agencias de financiamiento de investigación para publicar en revistas de corriente principal (Rogel-Salazar y otros, 2017), en las cuales los investigadores compiten por el prestigio académico e investigativo (De Moya-Anegón y otros, 2007). Por último, relacionar la producción científica, el tamaño de su fuerza de trabajo científica y la calidad científica de las publicaciones generan un punto de vista complementario a los análisis bibliométricos tradicionales y contextualizan la producción científica de los países con un ámbito socioeconómico similar (Garfield 1993; Rousseau y Rousseau 1998; Abramo y otros, 2015).

\section{REFERENCIAS}

Abramo, G.; D’Angelo, C. A.; Di Costa, F. (2015). A new approach to measure the scientific strengths of territories. Journal of the Association for Information Science and Technology, 66 (6), 1167-1177. https:// doi.org/10.1002/asi.23257

Acosta, O.; Celis, J. (2014). The emergence of doctoral programmes in the Colombian higher education system: Trends and challenges. Prospects, 44 (3), 463-481. https://doi.org/10.1007/s11125-014-9310-5

Aguado López, E.; Becerril García, A. (2016). Revista Investigación Económica: análisis bibliométrico a partir de redalyc.org, 2005-2014. Investigación Económica, 75 (295), 3-30. https://doi.org/10.1016/j.inveco.2016.03.001

Ardila, M. (2015). Colombia y México: hacia ¿diplomacias democráticas de baja intensidad? Desafíos, 27 (2), 221252. https://doi.org/10.12804/desafios27.2.2015.07

\section{CONCLUSIONES}

Colombia y México tienen semejanzas socioeconómicas, pero grandes diferencias en sus indicadores relativos de CyT. Entre 2006 a 2017, México tuvo un mayor presupuesto de investigación, una mayor fuerza laboral y produce una mayor cantidad de artículos por año. Colombia produce más artículos por doctor graduado, a pesar de tener menor presupuesto y menor mano de obra que México. Esto implica que Colombia es más eficiente que México en maximizar su producción científica por cada dólar de investigación invertido; sin embargo, en la mayoría de los casos, la producción científica mexicana se clasifica mejor que la colombiana. Los investigadores colombianos y mexicanos publican cada vez una mayor proporción de trabajos en revistas TOP, las cuales se favorecen de una mayor citación y visibilidad respecto a las revistas locales con mayor influencia que no se clasifican entre las revistas más prestigiosas del mundo. El uso de indicadores relativos en el análisis de la producción científica de Colombia y México complementa al análisis bibliométrico tradicional en el área de Ciencias Sociales.

\section{AGRADECIMIENTOS}

Este trabajo fue financiado por la Universidad Pedagógica y Tecnológica de Colombia (código SGI 1941). MRT agradece a Colciencias y Colfuturo (Beca para Estudios de Doctorado - 528).

\section{ACKNOWLEDGEMENTS}

This work was funded by the Universidad Pedagógica y Tecnológica de Colombia (code SGI 1941). MRT thanks Colciencias and Colfuturo (Scholarship for Doctoral Studies - 528).

Bornmann, L. ; Haunschild, R. (2016). Citation score normalized by cited references (CSNCR): The introduction of a new citation impact indicator. Journal of Informetrics, 10 (3), 875-887. https://doi.org/10.1016/j.joi.2016.07.002

Casas Guerrero, R. (2004). Conocimiento, tecnología desarrollo en América Latina. Revista Mexicana de Sociología, 66, Número especial, 255-277. https://doi. org/10.2307/3541453

Chinchilla-Rodríguez, Z.; Benavent-Pérez, M.; Moya-Anegón, F.; Miguel, S. (2012). International collaboration in medical research in Latin America and the Caribbean (2003-2007). Journal of the Association for Information Science and Technology, 63 (11), 2223-2238. https://doi.org/10.1002/asi.22669

Chinchilla-Rodríguez, Z.; Ocaña-Rosa, K.; VargasQuesada, B. (2016). How to combine research guarantor and collaboration patterns to measure scientific performance of countries in scientific fields: 
nanoscience and nanotechnology as a case study. Frontiers in Research Metrics and Analytics, 1 (2), 1-15. https://doi.org/10.3389/frma.2016.00002

Garfield, E. (1993). What citations tell us about Canadian research. Canadian Journal of Information and Library Science, 18 (4), 14-35.

Gómez N.; Jiménez A.; Guerrero. S.; Ayala Y. (2014). Análisis de la producción científica colombiana en química. Base de datos Wok (2001-2012). Revista Logos Ciencia \& Tecnología, 6 (1), 108-115. https://doi. org/10.22335/rlct.v6i1.125

Harzing, AW. (2019). Two new kids on the block: How do Crossref and Dimensions compare with Google Scholar, Microsoft Academic, Scopus and the Web of Science? Scientometrics, 120, 341-349. https://doi. org/10.1007/s11192-019-03114-y

Hofman, A.; Mas, M.; Aravena, C.; Fernández, J. (2017). Crecimiento económico y productividad en Latinoamérica. El proyecto LA-KLEMS. El trimestre económico, 84 (334), 259-306. https://doi.org/10.20430/ete.v84i334.302

Larivière, V.; Haustein, S.; Mongeon, P. (2015). The Oligopoly of Academic Publishers in the Digital Era. PLoS ONE, 10 (6), e0127502. https://doi.org/10.1371/ journal.pone. 0127502

Mansell, R.; Steinmueller, W.E. (2000). Mobilizing the Information Society: Strategies for Growth and Opportunity. Oxford: Oxford University Press.

Miguel, S.; Chinchilla-Rodríguez, Z.; Moya-Anegón, F. (2011). Open Access and Scopus: A New Approach to Scientific from the Standpoint of Access. Journal of the American Society for Information Science and technology, 62 (6), 1130-1145. http://doi.org/10.1002/asi.21532

Morales Gaitán, K. A.; Aguado López, E. (2010). La legitimación de la ciencia social en las bases de datos científicas más importantes para América Latina. Latinoamérica. Revista de Estudios Latinoamericanos, (51), 159-188.

Moya-Anegón, F. de; Chinchilla-Rodríguez, Z.; VargasQuesada, B.; Corera-Álvarez, E.; Muñoz-Fernández, F. J.; González-Molina, A.; Herrero-Solana, V. (2007). Coverage analysis of Scopus: A journal metric approach. Scientometrics, 73 (1), 53-78. https://doi. org/10.1007/s11192-007-1681-4

Pradhan, R.; Arvin, M.; Bahmani, S.; Bennett, S. (2017). The innovation- growth link in OECD countries: Could other macroeconomic variables matter? Technology in Society, 51, 113-123. https:// doi.org/10.1016/j.techsoc.2017.08.003

Pérez Reyes, G. A., y Martínez Rodríguez, A. (2014). La ciencia como empresa social: su evaluación desde la bibliometría. Biblios, 55, 27-39. https://doi. org/10.5195/biblios.2014.157

RICYT (2017). Red Iberoamericana e Interamericana de indicadores de Ciencia y Tecnología. Disponible en: http://www.ricyt.org/indicadores. [Fecha de consulta: 22/03/2019]
RICYT (2015). Red de indicadores de Ciencia y Tecnología, Iberoamericana e Interamericana. Disponible en: http://www.ricyt.org/indicadores. [Fecha de consulta: 15/05/2017]

Rodríguez-Gutiérrez, J.; Gómez-Velasco, N.; Herrera-Martínez, Y. (2017). Técnicas bibliométricas en dinámicas de producción científica en grupos de investigación. Caso de estudio: Biología-UPTC. Revista Lasallista de Investigación, 14(2), 73-82. https://doi.org/10.22507/rli.v14n2a7

Rogel-Salazar, R.; Santiago-Bautista, I.; Martínez-Domínguez N. (2017). Revistas científicas latinoamericanas de Comunicación indizadas en WoS, Scopus y bases de datos de Acceso Abierto. Comunicación y Sociedad, 30, 167-196. https://doi.org/10.32870/cys.v0i30.6514

Rousseau, S., y Rousseau, R. (1998). The scientific wealth of European nations: Taking effectiveness into account. Scientometrics, 42 (1), 75-87. https://doi. org/10.1007/BF02465013

SCImago. (2007). SJR - SCImago Journal \& Country Rank. Disponible en: http://www.scimagojr.com [Fecha de consulta: 20/04/2019]

Sancho, R. (1990). Indicadores bibliométricos utilizados en la evaluación de la ciencia y la tecnología. Revisión bibliográfica. Revista Española de Documentación Científica, 13 (3-4), 842-865.

Spinak, E. (1998). Indicadores cienciométricos. Ciência da informação, 27 (2), 141-148. https://doi. org/10.1590/S0100-19651998000200006

Teitel, S. (1994). Patents, R \& D Expenditures, Country Size, And Per-Capita Income: An International Comparison. Scientometrics, 29 (1), 137-159. https:// doi.org/10.1007/BF02018387

Teitel, S. (1985). Indicadores científico-tecnológicos: la América Latina, países industrializados y otros países en vía de desarrollo. El Trimestre Económico, 52 (205), 95-119.

Torres-Salinas, D.; Moreno-Torres, J.; Delgado-LópezCózar, E.; Herrera, F. (2011). A methodology for Institution-Field ranking based on a bidimensional analysis: the IFQ 2 A index. Scientometrics, 88 (3), 771-786. https://doi.org/10.1007/s11192-011-0418-6

UNESCO - The United Nations Educational, Scientific and Cultural Organization (2015). Informe de la UNESCO sobre la Ciencia: hacia 2030. Hechos y cifras. Disponible en: https://unesdoc.unesco.org/ark:/48223/ pf0000235407_spa [Fecha de consulta: 20/04/2019]

Van den Berg, H. (2016). Economic Growth and Development (Third Edition). Singapore: World Scientific Publishing Company. https://doi. org/10.1142/9058

Van Noorden, R. (2019). Indonesia tops open-access publishing charts. Nature. https://doi.org/10.1038/ d41586-019-01536-5

Villaveces Cardoso, J.; Orozco Castro, L.; Ruiz Ramos, C. ; Chavarro Bohórquez, D. ; Llanos Ballestas, E. ; Silva Bohórquez, A.; Herrera Herrera, B. (2007). La investi- 
gación en Uniandes 2006: una aproximación desde la cienciometría. Bogotá: Ediciones Uniandes.

Wang, Q.; Waltman, L. (2016). Large-scale analysis of the accuracy of the journal classification systems of Web of Science and Scopus. Journal of Informetrics, 10 (2), 347-364. https://doi.org/10.1016/j.joi.2016.02.003
Weick, K.; Caldera González, D.; Ortega Carrillo, M. (2009). Las organizaciones educativas como sistemas flojamente acoplados. Revista Gestión y Estrategia, (36), 93-110.

Witter, G. (1997). Produção científica. Campinas: Átomo. 